\title{
Betatron emission from relativistic electrons in a high intensity optical lattice
}

\author{
I. A. Andriyash, ${ }^{1,2, *}$ E. d'Humières, ${ }^{2}$ V. T. Tikhonchuk, ${ }^{2}$ and Ph. Balcou ${ }^{2}$ \\ ${ }^{1}$ Laboratoire d'Optique Appliquée, ENSTA-ParisTech, CNRS, Ecole Polytechnique, UMR 7639, 91761 Palaiseau, France \\ ${ }^{2}$ Université Bordeaux, CNRS, CEA, CELIA (Centre Lasers Intenses et Applications), UMR 5107, F-33400 Talence, France
}

(Received 30 May 2013; published 30 October 2013)

\begin{abstract}
We describe theoretically and numerically the interaction of a laser- or LINAC-accelerated beam of relativistic electrons with a high intensity optical lattice, resulting from the superposition of two transverse laser pulses. The bunch is trapped and guided within the potential channels of the optical lattice, leading to betatron oscillations. We describe the emission from individual particles and from the bunch, and analyze its spectrum, considering the dominant incoherent radiation as well as possible effects of partial coherency. Analysis of the emitted radiation should provide useful information on the characteristics of the electron beam, and its interaction with the optical lattice.
\end{abstract}

DOI: 10.1103/PhysRevSTAB.16.100703

PACS numbers: 41.60.Ap, 41.75.Jv, 42.65.Jx

\section{INTRODUCTION}

Numerous processes in laser-plasma interactions lead to generation of short wavelength radiation-from ultraviolet up to the gamma-ray range. Among these processes, increased attention is currently paid to the interaction between relativistic electron bunches, issued either from conventional accelerators, or from laser wakefield acceleration (LWFA) [1], and an intense laser light, that plays the role of an undulator.

Thomson, or inverse Compton scattering (ICS) is a well-known example, which leads to several technologies of fully polarized, quasimonochromatic x-ray beams, albeit with modest peak powers. For a typically short wavelength of the pump radiation, even moderately relativistic electrons scatter photons with the energies up to hundreds of kiloelectronvolts. This mechanism was observed in the scattering experiments of an infrared or free-electron laser off a bunch from a storage ring [2-5], head-on or $90^{\circ}$ scattering from a linear accelerator (LINAC) [6-8] or from LWFA electrons [9], coupling between a low-energy storage ring and a high finesse Fabry-Perot resonator [10-12].

In 2002, an alternative process was observed, when an ultrarelativistic bunch of electrons propagates through an underdense plasma and creates an ion channel. Relativistic electrons, oscillating in the focusing electrostatic field of this channel, emit short wavelength radiation [13]. The process was soon extended to the case of an ultrashort intense laser pulse as used in LWFA, which, propagating in a gas medium, creates a plasma wave, which copropagates with the pulse and is used to trap and to accelerate the electrons [14-16]. A strong incoherent

\footnotetext{
*igor.andriyash@gmail.com
}

Published by the American Physical Society under the terms of the Creative Commons Attribution 3.0 License. Further distribution of this work must maintain attribution to the author(s) and the published article's title, journal citation, and DOI. extreme-ultraviolet-radiation/x-ray emission was observed along the propagation direction of the accelerated electrons bouncing in the focusing electrostatic field of the plasma wave or ion channel $[17,18]$. By similarity with the betatron radiation from electrons in a guiding potential of linear accelerators [19], this radiation can be referred to as ion channel betatron emission (ICBE).

The spectral properties of emitted light are defined by the wiggler (undulator) strength parameter $K$, which is a ratio of electron trajectory angular deviation to the angular divergence of emitted light [20]. In ICS schemes, undulators are typically linear (wiggling the particle with a linearly polarized laser) and have a small strength $K \ll 1$, so that the emitted light is linearly polarized, and quasimonochromatic on axis. In contrast, recent studies of betatron emission in the LWFA $[18,21]$ have demonstrated that in the bubble regime of LWFA, the strength parameter may reach high values $K \sim 10$, which corresponds to a wiggler regime. The resulting $\mathrm{x}$ rays are nonpolarized, or partially polarized and have a broad synchrotron-type spectrum [22]. The axial and transverse accelerations in ICBE are intrinsically entangled, which leads to a time dependence of the $K$ parameter associated with each electron trajectory.

We have recently proposed another process of x-ray generation coupling relativistic electrons and intense laser pulses: the Raman x-ray free-electron laser [23,24]. The conceptual scheme is shown in Fig. 1. It implies overlapping of two identical laser pulses of the same frequency and polarization state to create an optical lattice at laser intensities high enough to induce the so-called strong field Kapitza-Dirac effect [25]. In these conditions, the laser ponderomotive potential forms a series of parallel valleys that can trap electrons in their transverse motion [26], and induces a bouncing along the optical lattice wave vector. The electrons emit light at two distinctively different frequency ranges: high frequency (x-ray) light by scattering the laser photons and low frequency light from the transverse bounce motion in the light lattice. The latter 


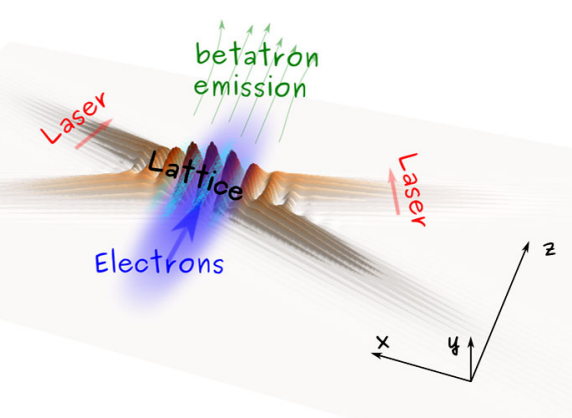

FIG. 1. The conceptual interaction scheme.

scheme has been considered before for a low intensity light and low-energy electrons emitting in the infrared range $[27,28]$.

In the present study, we propose to inject relativistic electrons in these high intensity transverse light wells, and examine the properties of the Doppler-shifted photon beam induced by the optical lattice betatron emission (OLBE). The radiation produced is typically in the UV domain, and its characteristics may provide useful information for the properties of the electron bunch and its interaction with the lattice.

After a summary of the typical laser and electron parameters expected to be used in this scheme, we first present a study of the dynamics of a single electron in the intense optical lattice, and, concentrating on the betatron emission, propose approximate formulas for the photon flux, spectrum, and the far field profile. Assuming a purely incoherent emission of the electron beam, we compare these results with the known characteristics of ICS and ICBE. We then turn to the analysis of a coherent radiation emission by a small size electron bunch. A general discussion and our concluding remarks are presented in the last section.

\section{INTERACTION PARAMETERS IN A HIGH INTENSITY OPTICAL LATTICE}

We first present a prospective setup that could generate betatron radiation from an electron beam with a relativistic factor $\gamma_{b} \sim 10^{2}$ in an intense optical lattice created with available lasers of a $10^{1}-10^{2} \mathrm{TW}$ power range. Higher energy electrons can also be considered with higher power lasers.

As shown in Fig. 2, we consider an electron bunch, issued either from a laser wakefield or a conventional linear accelerator, with kinetic energies of several tens of $\mathrm{MeV}$ and a normalized emittance ranging from 1 to a few $\mathrm{mm} \mathrm{mrad}$. The bunch impinges onto an optical lattice formed by the superposition of two identical laser pulses, synchronized in time and overlapping in space over the path of the electron bunch, in a line focus geometry, using either a homogeneous wave geometry $[29,30]$ or a grazing incidence (GRIP) geometry [31]. In the former case, the two laser pulses are normally incident onto the interaction

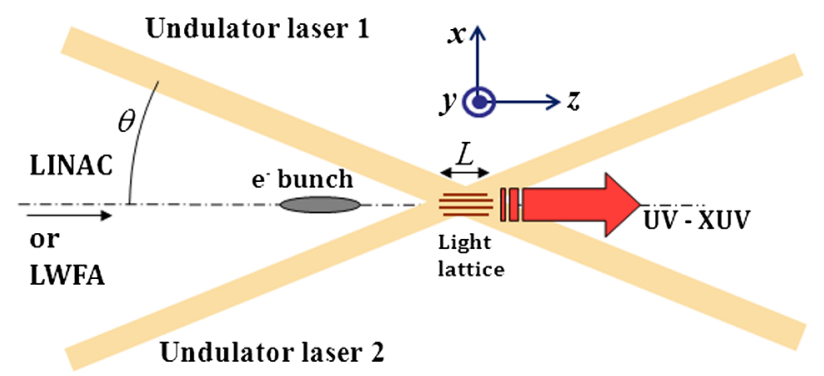

FIG. 2. Prospective experimental setup, with twin inhomogeneous laser beams impinging onto an interaction axis, and creating an intense optical lattice on the path of a relativistic electron bunch.

axis; in the latter, they are incident onto the interaction axis with an angle $\theta$, usually around $10^{\circ}$. This angle does not modify the depth of the ponderomotive potential channels, but allows one to vary the lattice period and thus to control the period of electron oscillations. We consider laser intensities in the line focus in the range from $10^{15}$ to $10^{18} \mathrm{~W} / \mathrm{cm}^{2}$ for near infrared lasers, resulting in nonrelativistic electron wiggling.

The necessary condition for the laser ponderomotive potential to trap a significant fraction of electrons was shown to read [26]

$$
\beta_{\perp}<\frac{\sqrt{2} a_{0}}{\gamma_{b}}
$$

where $v_{\perp}=\beta_{\perp} c$ is the root mean square (rms) transverse velocity of electrons in the interaction region, $c$ is the speed of light, $a_{0}$ is the normalized vector potential of either of the two transverse lasers, and $\gamma_{b}$ the average Lorentz factor of the relativistic electrons. The transverse velocity can be derived from the normalized bunch emittance $\epsilon^{N}$ as $v_{\perp}=$ $\epsilon^{N} / \gamma_{b} \sigma_{r}$, where $\sigma_{r}$ is the rms cross section of the bunch.

From (1), the laser intensity to be used is henceforth directly related to the average transverse velocities of the bunch in the interaction region. However, the typical values supplied by the two main technologies for electron acceleration, namely, conventional linear accelerators and laser wakefield acceleration, are clearly different, as are those of several basic parameters: bunch duration, cross sectional diameter, and total charge. In Table I, we present typical figures of such bunch parameters from the setups of the laser wakefield accelerator [32], and of the Thomsonscattering oriented linear accelerator in the X-band [34]. Linear accelerators provide typically pulses of picosecond durations that can be compressed down to below $1 \mathrm{ps}$. Such durations should be matched with picosecond intense laser technologies, based on amplifying media such as Nd:glass, or YAG. However, their typical divergence is low, allowing for moderately intense driving lasers. In contrast, electron bunches from the laser wakefield acceleration are extremely short, down to a few femtoseconds [32], and come out from the accelerating plasma with transverse 
TABLE I. Examples of system parameters.

\begin{tabular}{|c|c|c|}
\hline Accelerator & LWFA $[32,33]$ & LINAC [34] \\
\hline \multicolumn{3}{|c|}{ Electron beam } \\
\hline Energy & $84 \mathrm{MeV}$ & $45 \mathrm{MeV}$ \\
\hline Charge & $15 \mathrm{pC}$ & $0.7 \mathrm{nC}$ \\
\hline Spot size rms & $3 \mu \mathrm{m}$ & $27 \mu \mathrm{m}$ \\
\hline Duration rms & $1.5 \mathrm{fs}$ & $1 \mathrm{ps}$ \\
\hline Emittance & $1 \mathrm{~mm} \mathrm{mrad}$ & $1.41 \mathrm{~mm} \mathrm{mrad}$ \\
\hline \multicolumn{3}{|c|}{ Proposed laser specifications } \\
\hline Laser technology & Ti:sapphire & Yb:YAG [35] \\
\hline$\lambda_{0}$ & $0.8 \mu \mathrm{m}$ & $1.03 \mu \mathrm{m}$ \\
\hline Pulse duration & $30 \mathrm{fs}$ & $900 \mathrm{fs}$ \\
\hline Intensity & $1.2 \times 10^{17} \mathrm{~W} / \mathrm{cm}^{2}$ & $2 \times 10^{15} \mathrm{~W} / \mathrm{cm}^{2}$ \\
\hline $\begin{array}{l}\text { Laser energy over } \\
\qquad L=1 \mathrm{~mm}\end{array}$ & $0.21 \mathrm{~J}$ & $0.9 \mathrm{~J}$ \\
\hline \multicolumn{3}{|c|}{ Wavelength of scattered light for $\theta=90^{\circ}$} \\
\hline$\lambda_{s}$ & $35 \mathrm{~nm}$ & $67 \mathrm{~nm}$ \\
\hline \multicolumn{3}{|c|}{ Wavelength of scattered light for $\theta=10^{\circ}$} \\
\hline$\lambda_{s}$ & $204 \mathrm{~nm}$ & $384 \mathrm{~nm}$ \\
\hline
\end{tabular}

sizes in the micrometer range, implying much higher laser intensities to trap and wiggle the electrons. We can readily calculate the total laser energy, required to trap and wiggle the electron bunch over a characteristic distance $L=1 \mathrm{~mm}$. We assume that the laser is focused at normal incidence onto a line, whose width is equal to $2 \sigma_{r}$, readily attainable with $f / 10$ focusing optics. Table I shows that this amount is typically at the Joule level. It is lower for the titanium:sapphire technology, thanks to its short (femtosecond) pulse duration, better suited to the very short bunches of LWFA, than with picosecond pulses. However, ytterbium laser technology may also reach that energy level with compact laser systems.

It can be noted that the transverse dimension of the interaction region along the lattice axis is approximately $c \tau_{L} / 2 \sin \theta$, where $\tau_{L}$ is the laser pulse duration; typical value ranges are $c \tau_{L} \simeq 10 \mu \mathrm{m}$ for titanium sapphire technology, and $\simeq 300 \mu \mathrm{m}$ with $\mathrm{Yb}$ technologies. Both transverse directions are therefore large enough to ensure the robustness of the process with respect to fluctuations of the pointing of either electrons or photons. Another experimental issue is the timing fluctuations between the electron bunch and laser waves. In LWFA technology, the bunch duration and temporal jitter are clearly less than that of the driving LWFA laser; having both LWFA and optical lattice lasers derived from the same system should therefore grant a good synchronization. In LINAC technology, electron and photon durations should be approximately matched, and the timing jitter kept smaller than this value. The laser pulse duration can be increased to improve robustness with respect to this jitter, at the expense of increased laser energy. All these considerations will have to be revisited depending on each specific experimental implementation.

\section{SINGLE ELECTRON DYNAMICS IN THE OPTICAL LATTICE}

For the electron beam parameters in Table I, effects of space-charge can be considered as negligible, so that the orbit of each electron is defined by its initial phase coordinates and by the electromagnetic field. The two interfering laser beams, polarized along the $y$ axis and crossing with the angle $2 \theta$ (see Fig. 2), produce the following electromagnetic field potential:

$$
a_{y}=a_{1}+a_{2}=2 a_{0} \sin \left(\omega_{0} t-k_{\|} z\right) \sin \left(k_{\perp} x\right),
$$

where $\omega_{0}=2 \pi c / \lambda_{0}$ is the laser field frequency, and $\left(k_{\|}, k_{\perp}\right)=\omega_{0} / c(\cos \theta, \sin \theta)$ represent the longitudinal and transverse projections of laser wave vector onto the electron beam axis. The field amplitude is expressed as a vector potential, normalized in units of $m c^{2} / e$ and is defined numerically as $a_{0}=0.85 \times 10^{-9} \cdot \lambda_{0} \sqrt{I}$, where $I$ is the laser intensity measured in $\mathrm{W} / \mathrm{cm}^{2}$, and $\lambda_{0}$ is the laser wavelength in $\mu \mathrm{m}$.

The transverse distribution of electromagnetic field follows a standing wave pattern, with a wave number $k_{\perp}$; the ponderomotive potential due to this optical lattice has a sinusoidal dependence, and confines the electrons to a series of potential channels with the half-widths $\lambda_{0} /(4 \sin \theta)$. As was demonstrated in [23,28], in the ponderomotive potential of the interference of two laser waves, the small-amplitude electron oscillations have a bounce frequency:

$$
\Omega_{0}=\frac{\sqrt{2} a_{0} k_{\perp} c}{\gamma_{b}} .
$$

At the same time relativistic electrons oscillate along the laser polarization with a frequency $\omega_{0}^{\prime}=\omega_{0}\left(1-\beta_{z} \cos \theta\right)$, which is down-shifted due to the electron propagation at relativistic velocities along the laser wave (see Fig. 3).

For an arbitrary amplitude of electron oscillations, the frequency depends on the excursion $x_{\max }=$ $k_{\perp}^{-1} \arcsin \left(\beta_{x 0} \gamma_{b} / \sqrt{2} a_{0}\right)$, where $\beta_{x 0}$ is a maximum transverse velocity of the trapped particle. Defining the normalized excursion as $\xi=\beta_{x 0} \gamma_{b} / \sqrt{2} a_{0}$, we can find the frequency as

$$
\Omega=\Omega_{0} \frac{\pi}{2 \mathcal{K}\left(\xi^{2}\right)} \simeq \Omega_{0}\left(\frac{\sin \pi \xi}{\pi \xi}\right)^{1 / 6}
$$

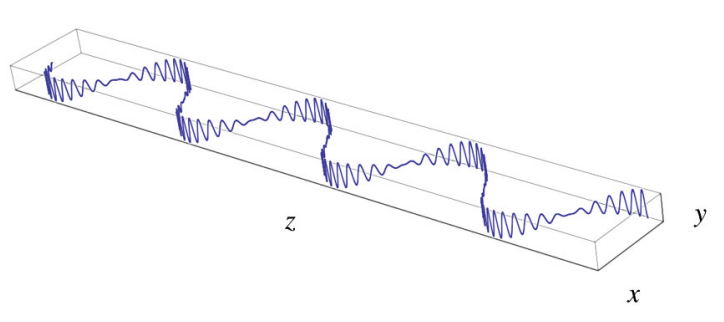

FIG. 3. Typical electron trajectory in an optical lattice. 
where $\mathcal{K}(m)=\int_{0}^{\pi / 2} d \theta\left(1-m \sin ^{2} \theta\right)^{-1 / 2}$ is an elliptic integral of the first kind, and the approximate expression has a high precision, $>99 \%$, for $\xi \in\{0,0.99\}$.

In order to compute the electron orbit, we make the assumption of a nonrelativistic transverse motion of electron, $a_{0} / \gamma_{b}, \beta_{x 0} \ll 1$, which is justified for the parameters discussed in the previous section. In what follows, we neglect the second order terms resulting from the variations of electron energy $\propto a^{2} / \gamma_{b}$, from the coupling of high and low frequency oscillation $\propto \Omega_{0} / \omega_{0}^{\prime}$, and we approximate the sinusoidal lattice potential with a parabolic one, $\sin ^{2} k_{\perp} x \simeq\left(k_{\perp} x\right)^{2}$. Considering the electron as initially located in the center of potential $\mathbf{r}_{0}=(0,0,0)$ with a velocity $\mathbf{v}_{0}=c\left(\beta_{x 0}, \beta_{y 0}, \beta_{z 0}\right)$, we derive its approximate trajectory as

$$
\begin{aligned}
\beta_{x} & =\beta_{x 0} \cos (\Omega t), \quad x_{e}=\left(\beta_{x 0} c / \Omega\right) \sin (\Omega t), \\
\beta_{y} & =\beta_{y 0}+\left(\sqrt{2} \Omega_{0} / c\right) x_{e} \sin \omega_{0}^{\prime} t, \\
y_{e} & =\beta_{y 0} c t-\left(\sqrt{2} \Omega_{0} / \omega_{0}^{\prime}\right) x_{e} \cos \omega_{0}^{\prime} t \\
\beta_{z} & =\beta_{z 0}-\left(\beta_{x}^{2}-\beta_{x 0}^{2}+\beta_{y}^{2}-\beta_{y 0}^{2}\right) / 2 \beta_{z 0} \\
z_{e} & =\beta_{z 0} c t
\end{aligned}
$$

Following the orbit Eq. (5), shown in Fig. 3, the electron emits two different electromagnetic waves. The first one, polarized along the $y$ axis, has a typical frequency $\omega_{s} \sim 2 \gamma_{b}^{2} \omega_{0}^{\prime}$ and represents the inverse Compton (Thomson) scattered laser light. The $x$-polarized field corresponds to the betatron emission and may be interpreted as the Doppler-shifted electron oscillation frequency, $\omega_{\beta} \sim 2 \gamma_{b}^{2} \Omega_{0}$. Introducing the wiggler strength as in [20], $K=\gamma_{b} \beta_{x 0} / \beta_{z 0}$, one may see that in the optical lattice this parameter is limited by the trapping condition Eq. (1), $K \lesssim \sqrt{2} a_{0}$. In the present study we assume moderate laser intensities, required to trap the majority of electrons, so that $K \ll 1$.

The angular-spectral distribution of emitted electromagnetic energy can be calculated using Lienard-Wiechert potentials $[17,20]$ :

$$
\frac{\mathrm{d} \mathcal{E}}{\mathrm{d} o \mathrm{~d} \omega}=\frac{e^{2}}{4 \pi^{2} c}\left|\int_{-\infty}^{\infty} \mathrm{d} t \frac{\mathbf{n} \times(\mathbf{n}-\boldsymbol{\beta}) \times \dot{\boldsymbol{\beta}}}{(1-\mathbf{n} \boldsymbol{\beta})^{2}} \mathrm{e}^{i \omega\left(t-\mathbf{n r}_{e} / c\right)}\right|^{2},
$$

where $\mathbf{r}_{e}, \boldsymbol{\beta}$, and $\dot{\boldsymbol{\beta}}$ are the position, velocity, and acceleration of the particle and $\mathbf{n}$ is a unit vector pointing in the observation direction. In terms of the spherical coordinates a unit vector to the observer reads

$$
\mathbf{n}=(\sin \zeta \cos \phi, \sin \zeta \sin \phi, \cos \zeta)
$$

Considering a finite length $L$ of the optical lattice, we may assume that the particle experiences no acceleration except for the interval $0<z<L$. Thus, we may replace the integral with infinite limits in Eq. (6), by the integration over the interval $t \in\left[0, t_{0}\right]$, where $t_{0}=L / c$ is the total interaction time.

The second factor in the integrand of Eq. (6) represents the emitted electromagnetic wave with a phase:

$$
\omega\left(t-\mathbf{n r}_{e} / c\right)=\hat{\omega} t-\left(\hat{k}_{x} x_{e}+\hat{k}_{y} y_{e}\right),
$$

where the first term defines the frequency of the wave in the electron frame, $\hat{\omega}=\omega\left(1-\beta_{z} \cos \zeta\right)$, and the second one accounts for its diffraction due to the electron motion along $x$ and $y$ axes, and is characterized by the transverse wave vector $\hat{\mathbf{k}}=(\omega / c) \mathbf{n}$. The phase factors proportional to the initial particle coordinate are discarded for a single particle case, but they are important for analysis of the coherence properties of the betatron emission of an electron beam (see the next section).

We consider only the betatron radiation, defined by the $x$ component of the integrand in Eq. (6) and assume the diffraction for this wave to be weak. Neglecting electron oscillations in the laser field and the second harmonic term $\beta_{x} \dot{\beta}_{x} \propto \sin (2 \Omega t)$, one may simplify Eq. (6):

$$
\begin{aligned}
\frac{\mathrm{d} \mathcal{E}}{\mathrm{d} o \mathrm{~d} \omega} \simeq & \frac{e^{2} \beta_{x 0}^{2}}{4 \pi^{2} c} \frac{\rrbracket_{0}^{2}\left(2 a_{0} \sin \zeta \cos \phi\right)}{(1-\mathbf{n} \boldsymbol{\beta})^{2}} \\
& \times\left(1-\frac{\sin ^{2} \zeta \cos ^{2} \phi}{1-\mathbf{n} \boldsymbol{\beta}}\right)^{2} G\left(N_{\Omega}, \frac{\omega}{\omega_{\beta}}\right),
\end{aligned}
$$

where we define the betatron radiation frequency $\omega_{\beta}=$ $\Omega /(1-\mathbf{n} \boldsymbol{\beta})$, and $N_{\Omega}=t_{0} \Omega / 2 \pi$ is the number of oscillations performed by the electron during the interaction time $t_{0}$. Here we denoted the Bessel function with $\rrbracket_{0}$ and the function

$$
G(n, w)=\left|\int_{0}^{2 \pi n} \mathrm{~d} \tau \sin (\tau) \mathrm{e}^{i \tau w}\right|^{2}
$$

can be simplified to trigonometric functions and calculated exactly.

Function $G\left(N_{\Omega}, \omega / \omega_{\beta}\right)$ defines a peak in the emitted spectrum at the frequency $\omega=\omega_{\beta}$ with a full width at half maximum (FWHM) $\Delta \omega / \omega_{\beta} \simeq 0.9 N_{\Omega}^{-1}$. This peak will be observed if the interaction parameters provide the condition $N_{\Omega} \gg 1$, which we consider satisfied in the further study. Considering the radiation emitted close to propagation axis $\zeta \ll 1$, one may derive a simplified factor of the relativistic Doppler shift:

$$
\frac{1}{1-\mathbf{n} \boldsymbol{\beta}} \simeq \frac{2 \gamma_{b}^{2}}{1+K^{2} / 2+\gamma_{b}^{2}\left[\beta_{y 0}^{2} \cos ^{2} \phi+\left(\zeta-\beta_{y 0} \sin \phi\right)^{2}\right]},
$$

where the terms $\propto \beta_{y 0}$ correspond to the shift along the $y$ axis of emission direction in the case of oblique propagation of the electron. For simplicity, in this section we put $\beta_{y 0}=0$, but this will be important for the study of emission from the bunch in Sec. IV. The central frequency emitted at $\zeta=0$ reads 


$$
\omega_{\beta 0}=\frac{2^{3 / 2} a_{0} \gamma_{b} \omega_{0} \sin \theta}{1+K^{2} / 2}\left(\frac{\sin \pi K / \sqrt{2} a_{0}}{\pi K / \sqrt{2} a_{0}}\right)^{1 / 6}
$$

The angular dependence in Eq. (7) integrated over the frequency describes the shape of the spot of emitted radiation. It is easy to show that, in the limit $K \ll 1$, the $y$ profile $(\phi=\pi / 2)$ of emission intensity distribution is $\propto\left(1+\zeta^{2} \gamma_{b}^{2}+K^{2} / 2\right)^{-3}$, and the one along the $x$ axis $(\phi=0)$ is $\propto\left(1+\zeta^{2} \gamma_{b}^{2}+K^{2} / 2\right)^{-7}$. Thus, the light is emitted into the elliptic spot stretched along the $y$ axis with the rms angular sizes:

$$
\Delta \zeta_{x}=\sqrt{2} /\left(5 \gamma_{b}\right) \sqrt{1+K^{2} / 2}, \quad \Delta \zeta_{y}=(5 / 3) \Delta \zeta_{x}
$$

Note that the increase of the emission angular size with the wiggler strength is accounted here by averaging over the betatron oscillations. A more accurate description, with account for the terms of higher infinitesimal order, may differ from Eq. (9), and it will be considered numerically.

Function $G(N, w)$ can be integrated in the spectral domain:

$$
\int_{0}^{\infty} \mathrm{d} w G\left(N_{\Omega}, w\right)=\pi^{2} N_{\Omega}\left(1-\frac{\sin \left(4 \pi N_{\Omega}\right)}{4 \pi N_{\Omega}}\right) \simeq \pi^{2} N_{\Omega},
$$

where the approximate expression stands for $N_{\Omega} \gg 1$. The further integration of Eq. (7) over the angles gives the full energy of betatron radiation. The result defines the number of photons with a frequency Eq. (8) emitted by a single electron as

$$
N_{\mathrm{ph}}=(7 \pi / 24) \alpha_{f} K^{2}\left(1+K^{2} / 2\right) N_{\Omega},
$$

where $\alpha_{f} \simeq 1 / 137$ is the fine-structure constant. The obtained expression is consistent with the result of a direct integration of Larmor's formula [17]. It is also useful to write emitted energy as a function of electron excursion for a given propagation length $z$ :

$$
\mathcal{E}(\xi) \simeq 8 e^{2} k_{0} a_{0}^{4} \sin ^{2} \theta \frac{z}{\lambda_{0}} \xi^{2}\left(\frac{\sin \pi \xi}{\pi \xi}\right)^{1 / 3} .
$$

The formulas [Eqs. (10) and (11)] are in a very good agreement, to within $2 \%$, with the result of numerical integration of Eq. (7).

A more rigorous verification of the presented findings may be performed by comparing them with the results of numerical simulations. In our analysis we use the numerical toolkit WIGGLER3D, which includes a three-dimensional particle tracker with electromagnetic pusher, various models of electromagnetic fields, including an optical lattice, and the module for calculation of coherent and incoherent spectra, using the formula given by Eq. (6).

We model the dynamics of an electron with $\gamma_{b}=100$ in an optical lattice formed by two laser beams with amplitudes $a_{0}=0.23$ directed with the angle $\theta=10^{\circ}$ to electron propagation. The particle is injected into the center of the lattice channel at various initial angles, and it propagates during a time of $6500 \lambda_{0} / c$, which provides about $N_{\Omega} \simeq 3$ betatron oscillations (exact number depends on excursion). In Fig. 4(a), we compare the total energy, in units of $e^{2} \omega_{0} / c$, as a function of particle excursion $\xi$, calculated numerically as an integral of the angular-spectral distribution (red dots), and the analytical estimate given by Eq. (11) (blue curve). Analytical and numerical estimates demonstrate a good agreement for a low excursion, while for $\xi \sim 1$ the electron oscillations become nonharmonic, which results in a slightly lower energy of emitted light.

A three-dimensional representation of emitted $x$-polarized spectrum and its integral projections $\left(\int \mathrm{d} \omega\right.$, $\int \mathrm{d} n_{x}$, and $\int \mathrm{d} n_{y}$ ) are shown in Fig. 4(b), for the excursion $\xi=0.8$. The bandwidth of the on-axis radiation $(\zeta=0)$, measured as a FWHM of the spectrum, agrees with the derived estimate $\Delta \omega_{\beta} / \omega_{\beta}=0.9 / N_{\Omega}=0.3$.

(a)

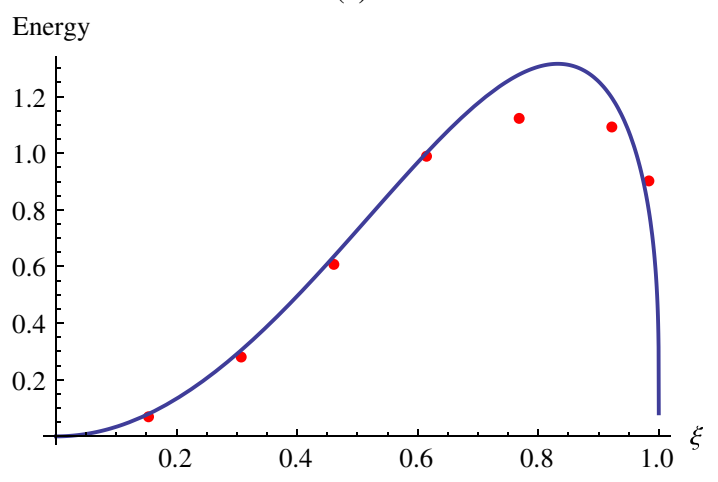

(b)

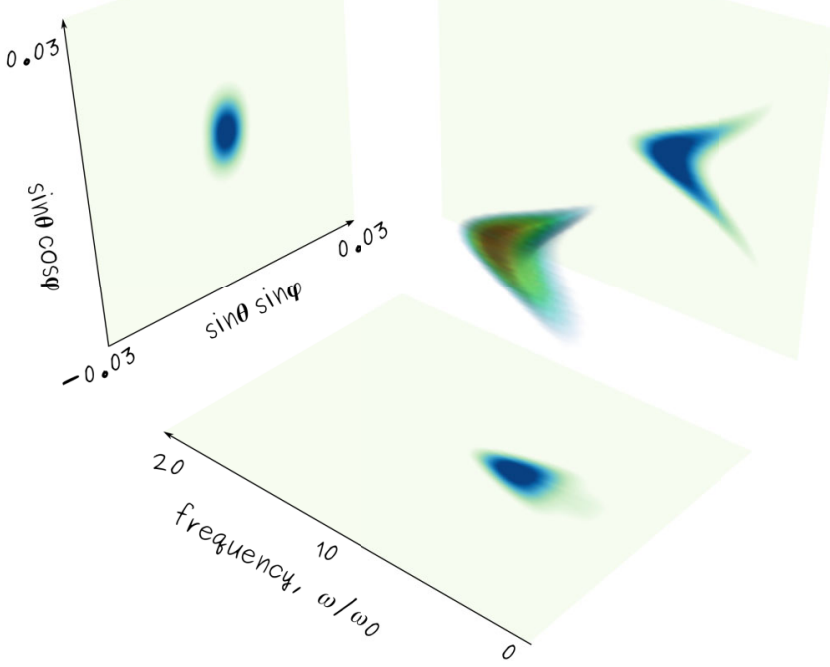

FIG. 4. Energy of $x$-polarized emission in the units of $e^{2} \omega_{0} / c$ as a function of electron excursion $\xi=\beta_{x} \gamma_{b} / \sqrt{2} a_{0}$ (a), calculated analytically (solid curve) and in numerical simulations (dots). Three-dimensional representation of emitted spectrum and its integral projections (b). 
As expected, the angular profile of emitted light is stretched in the plane $\phi=\pi / 2$. The dependences of emission angular sizes on the wiggler strength were studied by performing the series of simulations for a different $\beta_{x 0}$, and the results can be fitted as

$$
\begin{aligned}
& \Delta \zeta_{x} \simeq \sqrt{2} /\left(5 \gamma_{b}\right) \sqrt{1+K^{2}}, \\
& \Delta \zeta_{y} \simeq \sqrt{2} /\left(3 \gamma_{b}\right) \sqrt{1+(3 / 4) K^{2}} .
\end{aligned}
$$

These functions agree with Eq. (9) in the limit $K \rightarrow 0$, but demonstrate a faster growth of radiation divergence with $K$.

\section{BETATRON EMISSION FROM A BUNCH OF RELATIVISTIC ELECTRONS}

Let us now study the radiation produced by a macroscopic bunch of relativistic electrons trapped in an optical lattice, and find the relations between the characteristics of the bunch itself and those of the emitted light. Initially the bunch is characterized by the macroscopic parameters: duration $\tau_{b}$, transverse size $\sigma_{b}$, mean electron energy $\gamma_{b}$, and its normalized emittance $\epsilon^{N}=\sigma_{b} \gamma_{b} \beta_{\perp}$, where $\beta_{\perp}$ is a rms width of the distribution of electron transverse velocity. Interaction of the beam with the front edge of the optical lattice - the injection and trapping of the electrons in the ponderomotive potential channels-significantly modifies the electron distribution in the phase plane $\left(x, v_{x}\right)$, while the beam characteristics in the $Y$ and $Z$ phase planes are practically not affected.

The injection process is mainly defined by the length of the lattice ramp $l_{\text {ramp }}$ and the ratio $\gamma_{b} \beta_{0 \perp} / a_{0}$, and it was qualitatively described in [26]. It was shown that in the case of "fast" injection most of the particles with $x$ velocities satisfying the trapping condition [Eq. (1)] will become trapped in the lattice channels. The trapped particles will be distributed in the phase space $\left(x, \beta_{x}\right)$ region within the ellipse described by the energy conservation:

$$
\beta_{x}^{2}+2 a_{0}^{2} / \gamma_{b}^{2} \sin ^{2} k_{0 \perp} x<2 a_{0}^{2} / \gamma_{b}^{2} .
$$

The condition of fast injection here means that, on average, the electrons cross the ramp faster than they cross the channel half-width, which in our terms reads

$$
l_{\text {ramp }} \lesssim \lambda_{0} \sigma_{x} \gamma_{b} / 4 \epsilon^{N} \sin \theta .
$$

Since the electron energy is mainly defined by its motion along the $z$ axis, the energy spread $\Delta \gamma / \gamma$ may be considered as constant during the interaction.

One may calculate the radiation emitted by the electron bunch by considering the sum of Lienard-Wiechert vector potentials of each particle. The angular-frequency distribution of emitted energy can be described by Eq. (6), with the integrand presented as a sum of contributions from each electron. Neglecting the diffraction of emitted electromagnetic waves, we can write the distribution as

$$
\frac{\mathrm{d} \mathcal{E}_{\text {tot }}}{\mathrm{d} o \mathrm{~d} \omega}=\frac{e^{2}}{4 \pi^{2} c}\left|\sum_{j}^{N_{e}} \Phi_{0 j} \int_{0}^{t_{0}} \mathrm{~d} t \mathbf{A}_{j}\right|^{2},
$$

where the factor,

$$
\mathbf{A}_{j}=\frac{\mathbf{n} \times\left(\mathbf{n}-\boldsymbol{\beta}_{j}\right) \times \dot{\boldsymbol{\beta}}_{j}}{\left(1-\mathbf{n} \boldsymbol{\beta}_{j}\right)^{2}} \mathrm{e}^{i \hat{\omega} t},
$$

defines the amplitude of emitted field. The phase of emitted wave reads $\Phi_{0 j}=\exp \left(i \hat{\mathbf{k}} \cdot \mathbf{r}_{0 j}-\alpha_{j}\right)$, where $\mathbf{r}_{0 j}$ is the initial position of electron, and $\alpha_{j}$ is a phase of its oscillations.

Factor $\mathbf{A}_{j}$ only depends on the maximum of oscillating velocity of the $j$ th electron $\beta_{x 0}$, and the frequency of its betatron oscillations $\Omega$. To find the sum over the particles approximately, one may replace the amplitude of the field emitted by each particle by its average value $\left\langle\mathbf{A}_{j}\right\rangle$ and factor it out of the sum. Then, the expression for the total emitted energy reads

$$
\mathcal{E}_{\text {tot }}=\left|\sum \Phi_{0 j}\right|^{2} \cdot\langle\mathcal{E}\rangle,
$$

where $\langle\mathcal{E}\rangle$ is an average total energy emitted by a single electron.

To calculate the average of the energy emitted by electron in a beam, we need to consider the distribution of excursions of the trapped electrons and the dependence of electron emission energy on its excursion [Eq. (11)]. In a typical case, when trapped electrons occupy the full width of the lattice potential channel, their excursions are spread with a half-normal distribution $\left.\xi \sim \mathcal{N}\left(0, \sigma_{\xi}\right)\right|_{\xi>0}$ with a deviation $\sigma_{\xi} \simeq 0.5$. Therefore, the density of energy distribution as a function of excursion is $P(\xi) \propto$ $\left.\mathcal{E}(\xi) \mathcal{N}(0,0.5)\right|_{\xi>0}$. This allows one to calculate $\langle\mathcal{E}\rangle$ by integral averaging of $P(\xi)$ and it estimates

$$
\langle\mathcal{E}\rangle \simeq 2 e^{2} k_{0} a_{0}^{4}\left(L / \lambda_{0}\right) \sin ^{2} \theta .
$$

The presented findings are derived in a qualitative fashion, but they were verified by the numerical modeling and demonstrate good agreement for the wide range of interaction parameters. It is often convenient to characterize the scattering process with a value of its cross section $\sigma_{\beta}$, which is a ratio of the total emitted energy to a total flux of incident radiation. From Eq. (17), one may see that average power emitted by an electron in the lattice does not depend on the particle energy and can be estimated as

$$
P_{\beta} \simeq\left(e^{2} \omega_{0}^{2} / \pi c\right) a_{0}^{4} \sin ^{2} \theta .
$$

Considering the energy flux of two laser beams $I=$ $2 \times\left(c E_{0}^{2} / 4 \pi\right)$, we obtain the average cross section:

$$
\sigma_{\beta} \simeq(3 / 4 \pi) a_{0}^{2} \sin ^{2} \theta \sigma_{T},
$$

where $\sigma_{T}=(8 \pi / 3)\left(e^{2} / m c^{2}\right)^{2} \simeq 6.6 \times 10^{-25} \mathrm{~cm}^{2}$ is the Thomson cross section. 
Note that OLBE is defined by the trapping and wiggling of electrons in the ponderomotive potential in such a way that both oscillation frequency and excursion length are proportional to the amplitude of the lattice potential. The value of $\sigma_{\beta}$ depends therefore on the laser field, and is typically smaller than the Thomson cross section. It should be stressed that Eq. (19) does not incorporate any geometrical overlap factors, which have to be taken into account in each specific prospective experimental setup.

Function $P(\xi)$ also defines the weighted average excursion as $\langle\xi\rangle_{\mathcal{E}}=\langle\xi \mathcal{E}\rangle\langle\mathcal{E}\rangle^{-1} \simeq 0.6$ and its FWHM spread $\langle\Delta \xi\rangle_{\mathcal{E}} \simeq 0.6$. Physically, this means that most of the energy is emitted by the electrons with excursions $0.3<\xi<0.9$. This parameters will be important for the following study of emission bandwidth.

\section{A. Macroscopic incoherent emission from a bunch}

In the general case, when the electron oscillations can be considered as randomly phased, and/or the electron bunch is large enough $l_{x, y, z} \gg 2 \pi / \hat{k}_{x, y, z}$, the contributions of electrons can be considered as fully independent. Therefore, the sum Eq. (16) takes its minimal value and the total energy emitted incoherently estimates as

$$
\mathcal{E}_{\text {tot }}^{\text {incoh }} \simeq \sum\left|\Phi_{0 j}\right|^{2}\langle\mathcal{E}\rangle=N_{e}\langle\mathcal{E}\rangle
$$

The macroscopic parameters of the electron bunch (spatial profiles, emittance, and energy spread) define the angular-spectral characteristics of the emitted light, and its duration. Since emission frequency depends on the particle energy and excursion as described by Eq. (8), the emissions bandwidth is defined by variation of these parameters in the bunch and the number of electron oscillations. The total bandwidth may be approximately calculated as

$$
\frac{\Delta \omega_{\beta}}{\omega_{\beta}}=\sqrt{\frac{0.81}{N_{\Omega}^{2}}+\left(\frac{\partial \omega_{\beta}}{\partial \gamma_{b}} \frac{\Delta \gamma_{b}}{\omega_{\beta}}\right)^{2}+\left(\frac{\partial \omega_{\beta}}{\partial \xi} \frac{\Delta \xi}{\omega_{\beta}}\right)^{2}}
$$

where the first term is due to the broadening of a single particle spectrum. Assuming a quasiadiabatic injection, we consider the second term to be only defined by the initial energy spread of the particles, and it may be estimated as $\Delta \gamma_{b} / \gamma_{b}$.

The last term in Eq. (21) results from the spread of excursions of individual particles. From Eq. (8), one may write this term in a form

$$
\frac{\partial \omega_{\beta}}{\partial \xi} \frac{\Delta \xi}{\omega_{\beta}}=\left(\pi \xi \cot \pi \xi-1+\frac{12 a_{0}^{2} \xi^{2}}{1+a_{0}^{2} \xi^{2}}\right) \frac{\Delta \xi}{6 \xi},
$$

where for the trapped electron bunch, one may consider the values of $\langle\xi\rangle_{\mathcal{E}}$ and $\langle\Delta \xi\rangle_{\mathcal{E}}$ estimated earlier.

As we have shown in Sec. III, each electron emits into the spot with the sizes $\Delta \zeta_{x}, \Delta \zeta_{y}$, which is shifted along the $y$ axis by the angle $\zeta_{\text {shift }}=\beta_{y 0}$. In the case of a macroscopic electron beam, the finite emittance along the $y$ axis increases the angular size of emitted light as

$$
\left\langle\Delta \zeta_{y}\right\rangle=\sqrt{\Delta \zeta_{y}^{2}+\beta_{y}^{2}}
$$

The spot of emitted light is also "flattened" in the region $\zeta<\beta_{\perp} / \gamma_{b}$, so that the central frequency does not depend on $\zeta$. With help of Eq. (23), one may estimate the $y$ component of electron divergence from the measured angular size of emittance, thus, using OLBE as a beam diagnostic. This result is very important for the experimental realizations of OLBE.

Let us consider a beam of electrons with $\gamma_{b}=100$, rms values of cross-section radius and length $1.5 \lambda_{0}$, initial energy spread $\Delta \gamma_{b} / \gamma_{b}=0.03$, and beams angular divergence $2 \mathrm{~mm}$ mrad. We study the bunch propagation in a lattice of length $6500 \lambda_{0}$, with an injection ramp $l_{\text {ramp }}=$ $20 \lambda_{0}$, angle $\theta=10^{\circ}$, and $a_{0}=0.23$. We can model such an electron bunch numerically with a large number $2.5 \times 10^{3}$ of test particles. In Fig. 5(a) we show the particle trajectories during the injection and propagation phases. The observed fraction of the particles trapped in the central channel is around $43 \%$.

In Fig. 5(b), we present the angular-spectral distribution in the $\phi=\pi / 2$ plane of $x$-polarized light, where the white curve outlines the spectrum on axis, $\zeta=0$. The spectral distribution displays a peak at $\omega_{\beta}=8.7$ with a FWHM bandwidth $\Delta \omega_{\beta} / \omega_{\beta}=0.48$. The analytical estimate of the central frequency [Eq. (8) agrees with the calculated value for the $\langle\xi\rangle=0.77$, which is slightly different from the qualitative estimate $\langle\xi\rangle_{\mathcal{E}}=0.6$. Comparing the observed bandwidth with an estimate [Eq. (22)], one may find the value of excursion spread $\langle\Delta \xi\rangle \simeq 0.4$. With these values one may write the practically useful expression for the partial broadening of emitted spectrum due to the excursion spread:

$$
\Delta \omega_{\beta} / \omega_{\beta} \simeq 0.32+0.66 a_{0}^{2} .
$$

The angular distribution of emission is affected by the bunch emittance: the spectrum is flattened in a region $\zeta<\beta_{\perp} / \gamma_{b}$. The angular distribution of emission, integrated over the frequency, is shown in Fig. 5(c); it presents an elliptic shape, stretched along the $y$ axis similarly to the one produced by a single particle. Considering the series of initial velocity spreads $\beta_{y}$ we may verify the formula Eq. (23). In Fig. 6, the angular size of OLBE for $\phi=\pi / 2$, calculated from the simulations, is compared to the analytical expression and it demonstrates a good agreement.

The average energy emitted by a single electron in the simulations is $\langle\mathcal{E}\rangle=1.1 e^{2} k_{0}$, which agrees with the estimate Eq. (17). The corresponding average number of emitted photons estimates as $N_{\mathrm{ph}} \simeq 1.4 \times 10^{-3} / e^{-}$. For the parameters proposed for LWFA electrons, this estimate results in the emission of $\sim 10^{5}$ photons of $93 \mathrm{~nm}$ wavelength, which is well within reach of experimental detection techniques. 


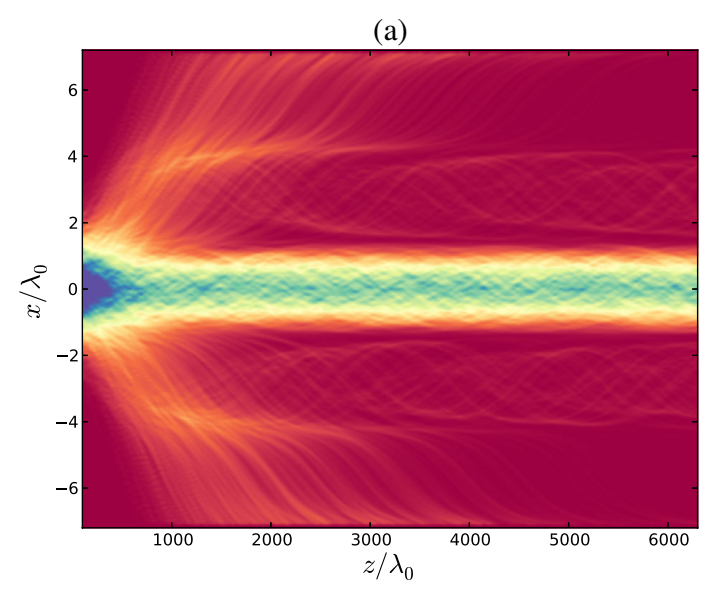

(b)

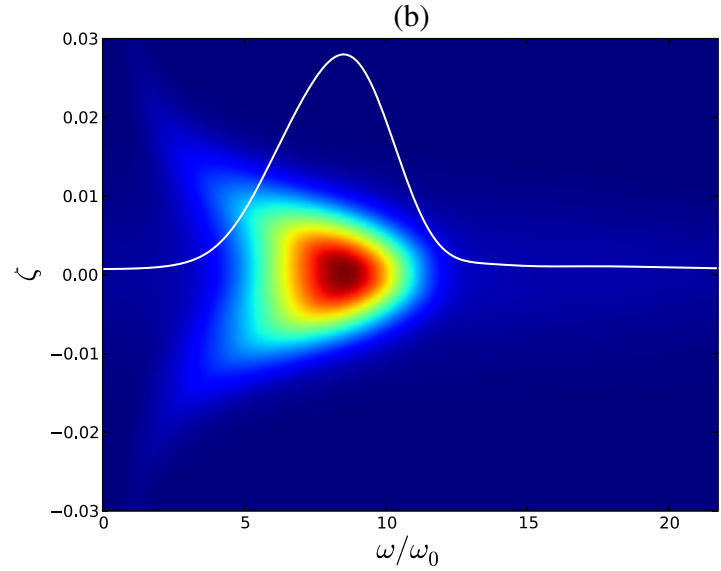

(c)

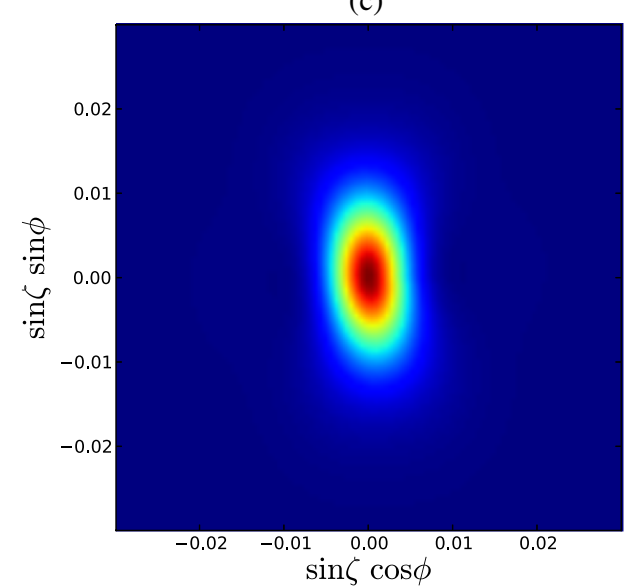

FIG. 5. Trajectories of electron propagation in the optical lattice, the length of injection zone is 20 wavelengths (a). Angular-spectral distribution of emitted radiation in the $\phi=\pi / 2$ plane and its on-axis spectrum (white curve) (b), and the frequency-integrated angular distribution (c).

\section{B. Partial coherence effects}

Most photon scattering processes in intense electromagnetic fields exhibit not only an incoherent, but also a coherent emission regime. Coherent inverse Compton

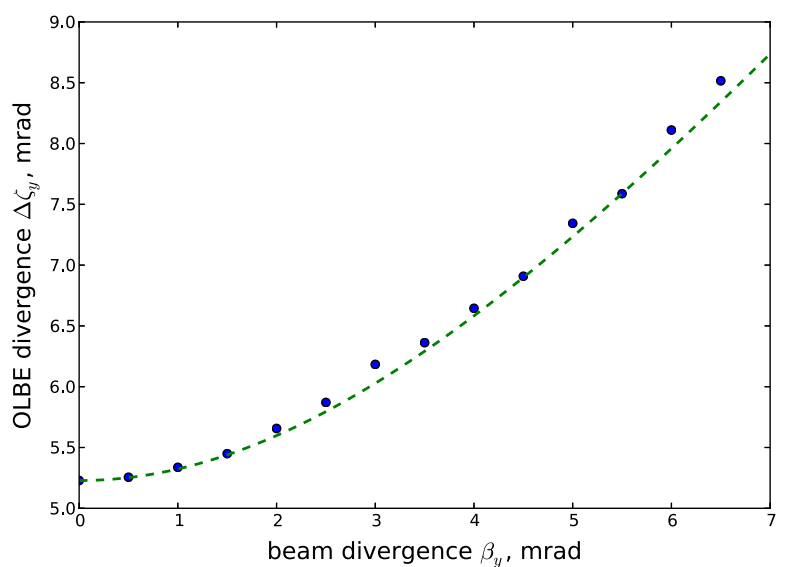

FIG. 6. Angular divergence of betatron emission in the nontrapped direction as a function of initial beam divergence. Blue dots show the simulation data and dashed curve corresponds to Eq. (23).

scattering has been considered as a prospective implementation of a laser-undulator free-electron laser. A similar situation is also well known in the physics of synchrotron radiation, with the process of coherent synchrotron radiation (CSR). If the bunch length is short enough to be comparable to the wavelength of part of the synchrotron spectrum, then the individual emissions interfere constructively, and the total emitted radiation results from the coherent superposition of all electron contributions, implying a major flux enhancement at those wavelengths. Coherent synchrotron radiation was observed for the first time by Nakazato and co-workers in 1990 [36] in the far infrared domain. It since proved very rich of information on the bunch dynamics, providing evidence of multiple bunches, or of microbunching phenomena [37], and has to be taken into account in the design of modern light sources. In [38], Chubar has provided a comprehensive survey of the basic formula and numerical methods of CSR.

One may note an analogy between CSR and betatron emission in an optical lattice, in the case of a short electron bunch, as issued from laser wakefield. The bunch duration of a few femtoseconds is comparable or smaller than the typical period of betatron oscillations, so that a collective displacement of the bunch appears possible. Let us investigate therefore the situation when the contributions to betatron emission in the optical lattice of all individual electrons may become partially or fully coherent. For this, assuming a large number of particles, we write the phases term in Eq. (16) as

$$
\sum_{j}^{N_{e} \gg 1} \Phi_{0 j} \simeq \int \mathrm{d} \mathbf{r} n_{e}(\mathbf{r}) \exp [i \hat{\mathbf{k}} \mathbf{r}-\alpha(\mathbf{r})],
$$

where $n_{e}(\mathbf{r})$ is an electron density distribution and $\alpha(\mathbf{r})$ is a distribution of the phase of betatron oscillations. The transverse components of the emitted light wave vector are small and may be neglected, $\hat{k}_{x, y} \ll \hat{k}_{z} \simeq 2 \gamma_{b}^{2} \Omega / c$. 
Considering a modulation of electron density or oscillation phase with a wavelength $\mathbf{k}_{\text {mod }} \simeq \hat{\mathbf{k}}$, the sum in Eq. (25) is equal to the number of electrons $N_{e}$, and the total emitted energy is $\mathcal{E}_{\text {tot }}^{\mathrm{coh}} \simeq N_{e}^{2}\langle\mathcal{E}\rangle$. This corresponds to the free-electron-laser-type coherent emission, which is realized in the free-electron laser facilities either by injecting a copropagating resonant "seed" wave, or in the regime of self-amplified spontaneous emission (SASE), due to the parametric instabilities.

CSR-type coherency is also possible, if two conditions are met: (i) the longitudinal size of the bunch is small $z \hat{k}_{z}<1$, and (ii) if electrons oscillate in a synchronized fashion, so that $\alpha(\mathbf{r}) \simeq$ const. For synchronized oscillations the phase in Eq. (25) can be discarded, and it represents the Fourier image of the particle density distribution at $\mathbf{k}=\hat{\mathbf{k}}$. For a beam with a Gaussian temporal profile, the emitted energy is

$$
\mathcal{E}_{\text {tot }}^{\text {sync }} \simeq\langle\mathcal{E}\rangle N_{e}^{2} \exp \left(-\omega_{\beta}^{2} \tau_{b}^{2}\right),
$$

where $\tau_{b}$ is the rms bunch duration. One may further evaluate Eq. (26) to obtain the amplification condition, $\mathcal{E}_{\text {tot }}^{\text {sync }} /\langle\mathcal{E}\rangle N_{e}>1$, which leads to

$$
\tau_{b} c / \lambda_{0} \lesssim \frac{0.1}{a_{0} \gamma_{b} \sin \theta} \sqrt{\log _{10} N_{e}}
$$

For the case of the optical lattice designed for the scattering of LWFA electrons (see Table I), the electron bunch is of the charge of a few tens of $\mathrm{pC}$ and mean energy $\gamma_{b}=100$. Then, Eq. (27) indicates a possible coherent emission from the electron current inhomogeneities at the subfemtosecond or attosecond level $\lesssim 0.08 \lambda_{0} / c$. This condition shows that ultrashort substructures within LWFA bunches might be detected by this method, if their duration is of the order of one-tenth of the bunch duration diagnosed by Lundh et al. [32], which, while challenging, is within the range of physical possibilities.

Condition (i) on the bunch, or sub-bunch, duration is not however sufficient: condition (ii) requires a collective betatron oscillation. In practice, such a collective oscillation can be imposed by inducing optically a kick on the bunch by side shifting the lattice axis during the beam propagation, or by changing its direction-both methods needing only to control the spatial phase of one of the twin laser beams. Injecting the bunch into the lattice with an angle also results in a collective excitation, if the ramp-up region is short enough - in the opposite case, a long ramp would simply result in an adiabatic adaptation of the electron beam to the lattice, with a higher temperature and zero mean transverse values.

An important effect, related to the collective particle oscillations in a potential of a sinusoidal shape, is a kinetic mixing of electron orbits due to the fact that the oscillation frequency depends on the excursion length, according to Eq. (4). In [26] it was shown that kinetic mixing leads to a damping of the collective oscillations during the time

$$
t_{\mathrm{damp}}=\left\langle\tau_{\mathrm{osc}}^{2} /\left(2 L \partial_{x} \tau_{\mathrm{osc}}\right)\right\rangle .
$$

For a high excursion of trapped electrons, this period is estimated as $t_{\text {damp }} \simeq 0.84 \tau_{\text {osc }}$, which means that in order to maintain the coherent emission regime one should

(a)

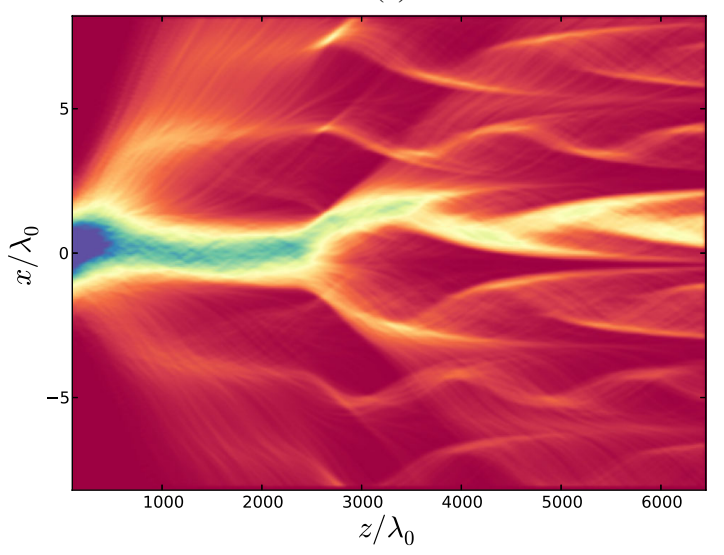

(b)

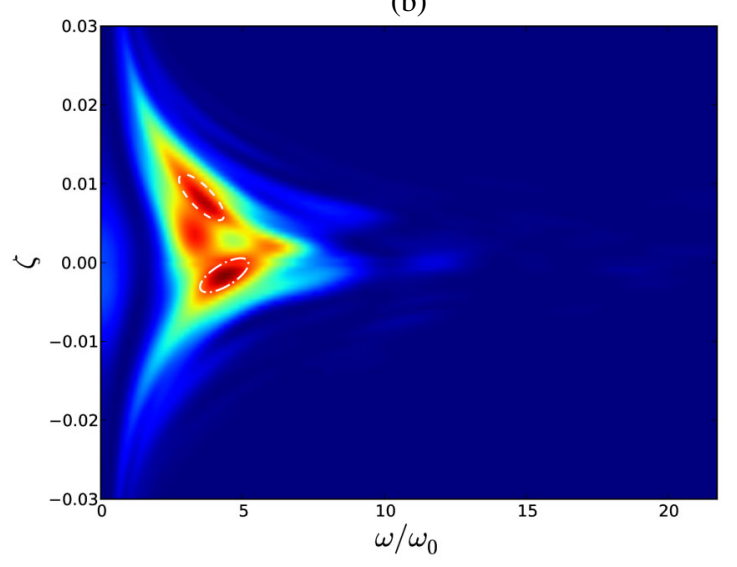

(c)

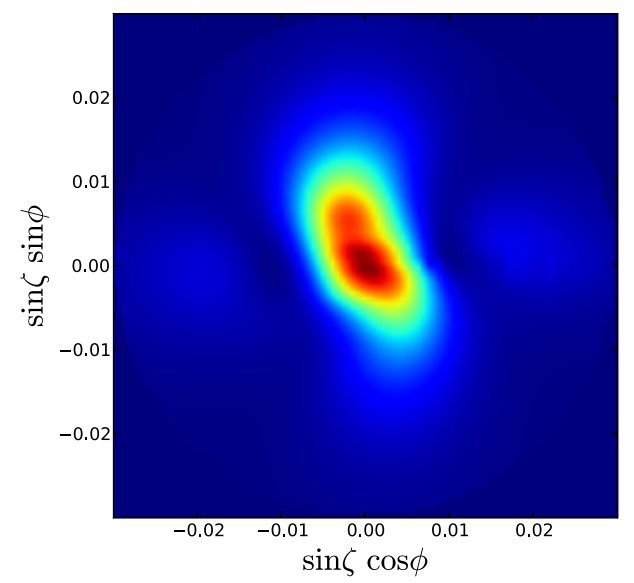

FIG. 7. Propagation of electrons injected obliquely into the optical lattice, followed by a KICK (a). Angular-spectral distribution of coherently emitted energy in the $\phi=\pi / 2$ plane (b) and its angular distribution integrated over the frequency (c). 
introduce the synchronization approximately every period of oscillations.

As a numerical confirmation, we calculate the spectrum of coherent emission using a large number of test particles, $N_{e} \sim 10^{3}-10^{4}$. The beam is modeled with the same parameters as in the previous section and duration $\tau_{b}=$ $0.057 \lambda_{0} / c$, which nearly satisfies Eq. (27). The electrons are injected and propagate in a lattice of length $6500 \lambda_{0}$, with a ramp of $50 \lambda_{0}$ and amplitude $a_{0}=0.23$. The electron synchronization is induced by the oblique injection of electrons at the angle $1.5 \mathrm{mrad}$, and by the kick placed at the distance of one oscillation period, $2300 \lambda_{0}$, from the lattice entrance. The kick of the $15 \lambda_{0} z$ length is modeled as a linear side shift of the lattice channels by the distance $0.7 \pi / 2 k_{\perp}$ along the $x$ axis.

In Fig. 7(a), one may see the particle trajectories for the case, where synchronization is first due to the oblique injection of the beam and is later enhanced by the kick. The fraction of particles, which are trapped in a central potential channel is around 25\%. Animated dynamics of the particle oscillations in $(z, x)$ and $\left(x, p_{x}\right)$ phase planes is presented in the supplemental materials [39]. Note that both synchronization mechanisms provide only partial phasing of electron oscillations, which results in a partial coherency, also affected by the spread of particle energy and excursion. Thus, a theoretical estimate of the coherent amplification can hardly be performed accurately, and the formula Eq. (26) should be taken qualitatively.

The angular-spectral distribution of coherent emission from the twice synchronized electrons is presented in Fig. 7(b) for $\phi=\pi / 2$. The distribution has two distinctive peaks, where the one at $\zeta=7 \mathrm{mrad}$ is due to the oblique injection (dashed ellipse) and the second one at $\zeta=-1.4 \mathrm{mrad}$ (dot-dashed ellipse) results from the kick.
The latter peak is more intense and it results into the peak in the angular distribution shown in Fig. 7(c).

If compared to the case of nonsynchronized electron oscillations, the emitted energy is enhanced by the factor $\mathcal{E}^{\mathrm{coh}} /\langle\mathcal{E}\rangle \simeq 16$, around $80 \%$ results from the kick. The frequency of the peaks is down-shifted by the factor $\sim\left(1+\zeta \gamma_{b}\right)^{-3}$, due to the angular dependence of the relativistic Doppler effect.

\section{DISCUSSION AND SUMMARY}

Let us now recapitulate the main properties and scaling laws of the optical lattice betatron emission (OLBE) with respect to macroscopic parameters of the interaction. It is instructive to compare qualitatively OLBE with three similar processes: inverse Compton scattering (ICS), ion channel betatron emission (ICBE), optical transition radiation (OTR). In Table II we summarize the formulas derived in our study and present them in a simplified ready-to-use form.

Similarly to other scattering schemes, the duration of generated radiation is defined by the duration of driver electron bunch. Propagating the distance $L$ the scattered photons overtake the electrons, thus stretching the emitted pulse as

$$
\tau_{\beta} \simeq \tau_{b}+L /\left(2 \gamma_{b}^{2} c\right) .
$$

For a submillimeter interaction length and/or high electron energies this effect is negligible, however, it may become significant for the OLBE with the discussed parameters.

Similarly to inverse Compton scattering, OLBE is fully polarized along the lattice optical axis. In contrast to ICS, the angular distribution is not isotropic in the transverse plane; we predict a slightly elliptic angular distribution, arising from existence of an intrinsic orientation along the

TABLE II. OLBE formulas.

\begin{tabular}{|c|c|}
\hline $\begin{array}{l}\text { Electron trapping condition } \\
\text { Electron oscillation length } \\
\text { Emission cross section } \\
\text { Emission wavelength }\end{array}$ & $\begin{array}{c}\beta_{\perp}<\sqrt{2} a_{0} / \gamma_{b} \\
L_{\beta}=1.1 a_{0} \sin \theta / \gamma_{b} \\
\sigma_{\beta}=(3 / 4 \pi) a_{0}^{2} \sin ^{2} \theta \sigma_{T} \\
\lambda_{\beta}=L_{\Omega}\left(1+0.6 a_{0}^{2}\right) /\left(2 \gamma_{b}^{2}\right)\end{array}$ \\
\hline \multicolumn{2}{|l|}{ Emission angular divergence (rms) } \\
\hline Trapped & $\Delta \zeta=\sqrt{2} /\left(5 \gamma_{b}\right)\left(1+1.2 a_{0}^{2}\right)^{1 / 2}$ \\
\hline Nontrapped & $\Delta \zeta_{y}=\gamma_{b}^{-1}\left(2 / 9+0.2 a_{0}^{2}+\beta_{\perp 0}^{2} \gamma_{b}^{2}\right)^{1 / 2}$ \\
\hline Emission bandwidth (FWHM) & $\frac{\Delta \omega_{\beta}}{\omega_{\beta}}=\left\{\left(0.32+0.66 a_{0}^{2}\right)^{2}+0.81\left(L_{\Omega} / L\right)^{2}+5.45\left(\Delta \gamma_{b} / \gamma_{b}\right)^{2}\right\}^{1 / 2}$ \\
\hline Condition for coherent emission & $\tau_{b} c / \lambda_{0} \lesssim 0.24 \lambda_{\beta} \sqrt{\log _{10} N_{e}}$ \\
\hline \multicolumn{2}{|l|}{ Used notations: } \\
\hline Laser amplitude & $a_{0}=0.85 \lambda_{0}[\mu \mathrm{m}] \sqrt{I_{\mathrm{las}}\left[10^{18} \mathrm{~W} / \mathrm{cm}^{2}\right]}$ \\
\hline Electron Lorentz factor & $\gamma_{b}=\varepsilon_{e}[\mathrm{MeV}] / 0.511$ \\
\hline Number of electrons & $N_{e}=Q_{b} / e$ \\
\hline Thomson cross section & $\sigma_{T}=6.6 \times 10^{-25} \mathrm{~cm}^{2}$ \\
\hline
\end{tabular}


axis of the optical lattice. The angular divergence is of the order of the inverse Lorentz factor; and the emission presents the same angular chirp as ICBE, with decreasing frequencies for increasing angular deviations from the central axis.

The spectral characteristics put OLBE in an intermediate position between ICS and ICB. The former usually displays a rather narrow spectrum on the axis; the latter yields a very broad spectrum. From our analysis and numerical tests, the relative spectral width of OLBE is expected to be around $30 \%$, making it a broadband, but not white, photon source. The major difference between OLBE and ICS concerns the photon energy. In ICS, the photon energy scales as the square of the Lorentz factor $\gamma_{b}$, whereas it scales linearly with $\gamma_{b}$ in OLBE, resulting in a typical frequency range extending from the visible (for grazing incidence geometries) up to the extreme UV. This $\gamma_{b}$ dependence is typical for the betatron emission [1].

Because of the small cross section, the expected output photon flux of OLBE is rather low, about $10^{5}$ photons per shot. Not being practically interesting as a photon source by itself, this scheme provides information concerning the relativistic electron beam and the high intensity optical lattice. A number of diagnostic techniques based on the scattering processes have already being developed including betatron [40,41] and Compton [6,42] spectroscopy, transition radiation $[32,43]$, and quadrupole scan [44]. In particular, the setup for OLBE resembles the Shintake monitor [42], but is principally different involving the low-energy emission from the trapped electrons.

In Sec. IVA we have demonstrated how OLBE may be used to estimate the angular divergence of electron beam along the selected direction. From the spectral characteristics of OLBE the bunch duration and the energy spread may be retrieved similarly to OTR. Moreover, the OLBE can be very sensitive to small scale substructures within the bunch, which might lead to a partially coherent emission and affect the angular distribution of emitting light. This partially coherent emission can be stimulated by inducing a kick, or a shift of the optical lattice-namely, modifying the positions of the intensity minima. From an experimental point of view, the parameters of such a kick (position, lateral displacement) can be controlled by optical means, such as a deformable mirror. The resulting enhancement was shown to depend on the spatial size of the electron substructures. This allows one to estimate, for example, the effect of kinetic mixing, and hence estimate electron beam phase space distribution and its dynamics.

The OLBE can be implemented with a variety of geometrical or laser conditions, depending on laser intensity, interaction length, crossing angle $\theta$ between the optical lattice twin lasers, etc. Thanks to the flexibility of the setup, OLBE can complement the existing diagnostics providing more information on the electron bunch, a crucial point in view of the numerous upcoming applications of laser-accelerated electron beams. This technique may also be used to infer the interaction conditions in view of triggering or optimizing the Raman x-ray free-electron laser process [24].

[1] S. Corde, K. Ta Phuoc, G. Lambert, R. Fitour, V. Malka, A. Rousse, A. Beck, and E. Lefebvre, Rev. Mod. Phys. 85, 1 (2013).

[2] R. W. Schoenlein, W. P. Leemans, A. H. Chin, P. Volfbeyn, T. E. Glover, P. Balling, M. Zolotorev, K.-J. Kim, S. Chattopadhyay, and C. V. Shank, Science 274, 236 (1996).

[3] V. N. Litvinenko et al., Phys. Rev. Lett. 78, 4569 (1997).

[4] A. D’Angelo, O. Bartalini, V. Bellini, P. Levi Sandri, D. Moricciani, L. Nicoletti, and A. Zucchiatti, Nucl. Instrum. Methods Phys. Res., Sect. A 455, 15 (2000).

[5] K. Kawase, Y. Arimoto, M. Fujiwara, S. Okajima, M. Shoji, S. Suzuki, K. Tamura, T. Yorita, and H. Ohkuma, Nucl. Instrum. Methods Phys. Res., Sect. A 592, 154 (2008).

[6] W. P. Leemans, R. W. Schoenlein, P. Volfbeyn, A. H. Chin, T. E. Glover, P. Balling, M. Zolotorev, K. J. Kim, S. Chattopadhyay, and C. V. Shank, Phys. Rev. Lett. 77, 4182 (1996)

[7] D. J. Gibson et al., Phys. Plasmas 11, 2857 (2004).

[8] F. Albert et al., Phys. Rev. ST Accel. Beams 13, 070704 (2010).

[9] H. Schwoerer, B. Liesfeld, H.-P. Schlenvoigt, K.-U. Amthor, and R. Sauerbrey, Phys. Rev. Lett. 96, 014802 (2006).

[10] Z. Huang and R. D. Ruth, Phys. Rev. Lett. 80, 976 (1998).

[11] C. Bruni, N. Artemiev, R. Roux, A. Variola, F. Zomer, and A. Loulergue, UVX 2010-10e Colloque sur les Sources Cohérentes et Incohérentes UV, VUV et X; Applications et Développements Récents (2011), pp. 49-55.

[12] A. Variola, A. Loulergue, and F. Zomer, ThomX Conceptual Design Reports No. LAL RT 09/28 and No. SOLEIL/SOU-RA-2678, 2009.

[13] S. Wang et al., Phys. Rev. Lett. 88, 135004 (2002).

[14] S. P. D. Mangles et al., Nature (London) 431, 535 (2004).

[15] C. G. R. Geddes, Cs. Toth, J. van Tilborg, E. Esarey, C. B. Schroeder, D. Bruhwiler, C. Nieter, J. Cary, and W.P. Leemans, Nature (London) 431, 538 (2004).

[16] J. Faure, Y. Glinec, A. Pukhov, S. Kiselev, S. Gordienko, E. Lefebvre, J.-Ph. Rousseau, F. Burgy, and V. Malka, Nature (London) 431, 541 (2004).

[17] E. Esarey, B. A. Shadwick, P. Catravas, and W.P. Leemans, Phys. Rev. E 65, 056505 (2002).

[18] K. Ta Phuoc, F. Burgy, J. P. Rousseau, V. Malka, A. Rousse, R. Shah, D. Umstadter, A. Pukhov, and S. Kiselev, Phys. Plasmas 12, 023101 (2005).

[19] Martin Reiser, Theory and Design of Charged Particle Beams (Wiley-VCH, New York, 2008), 2nd ed.

[20] J.D. Jackson, Classical Electrodynamics (Wiley-VCH, New York, 1998), 3rd ed.

[21] S. Kiselev, A. Pukhov, and I. Kostyukov, Phys. Rev. Lett. 93, 135004 (2004).

[22] S. Fourmaux et al., New J. Phys. 13, 033017 (2011).

[23] Ph. Balcou, Eur. Phys. J. D 59, 525 (2010).

[24] I. A. Andriyash, E. d'Humières, V. T. Tikhonchuk, and Ph. Balcou, Phys. Rev. Lett. 109, 244802 (2012). 
[25] P. H. Bucksbaum, D. W. Schumacher, and M. Bashkansky, Phys. Rev. Lett. 61, 1182 (1988).

[26] I. A. Andriyash, Ph. Balcou, and V. T. Tikhonchuk, Eur. Phys. J. D 65, 533 (2011).

[27] S. Sepke, Y. Y. Lau, J.P. Holloway, and D. Umstadter, Phys. Rev. E 72, 026501 (2005).

[28] M. V. Fedorov, K. B. Oganesyan, and A. M. Prokhorov, Appl. Phys. Lett. 53, 353 (1988).

[29] J.-C. Chanteloup et al., J. Opt. Soc. Am. B 17, 151 (2000).

[30] L. Charreyre, S. Sebban, and Ph. Balcou (to be published).

[31] R. Keenan, J. Dunn, P. K. Patel, D. F. Price, R. F. Smith, and V. N. Shlyaptsev, Phys. Rev. Lett. 94, 103901 (2005).

[32] O. Lundh et al., Nat. Phys. 7, 219 (2011).

[33] S. Kneip et al., Nat. Phys. 6, 980 (2010).

[34] C. Tanga et al., Nucl. Instrum. Methods Phys. Res., Sect. A 608, S70 (2009).
[35] C. Wandt, S. Klingebiel, M. Siebold, Z. Major, J. Hein, F. Krausz, and S. Karsch, Opt. Lett. 33, 1111 (2008).

[36] T Nakazato et al., Phys. Rev. Lett. 63, 1245 (1989).

[37] J. M. Byrd, W.P. Leemans, A. Loftsdottir, B. Marcelis, M. C. Martin, W. R. McKinney, F. Sannibale, T. Scarvie, and C. Steier, Phys. Rev. Lett. 89, 224801 (2002).

[38] O. Chubar, Infrared Phys. Technol. 49, 96 (2006).

[39] See Supplemental Material at http://link.aps.org/ supplemental/10.1103/PhysRevSTAB.16.100703 for animated dynamics of the particle oscillations.

[40] M. Schnell et al., Phys. Rev. Lett. 108, 075001 (2012).

[41] G. R. Plateau et al., Phys. Rev. Lett. 109, 064802 (2012).

[42] T. Shintake, Nucl. Instrum. Methods Phys. Res., Sect. A 311, 453 (1992).

[43] C. Lin et al., Phys. Rev. Lett. 108, 094801 (2012).

[44] R. Weingartner et al., Phys. Rev. ST Accel. Beams 15, 111302 (2012). 